\title{
Public Health Genomics
}

Agapidaki, E. 153

Aggarwal, A. 132

Balling, R. 160

Brall, C. 178

Brand, A. 187

Burgunder, J.M. 170

Cronin, M. 160

Dunlop, J. 170

Esposito, G. 170

French, D. 132

Glorioso, V. 137

Gorwood, P. 170

Hasardzhiev, S. 153

Henderson, R. 132

Hermansson, L.-L. 137

Hopley, C. 137

Horgan, D. 129, 144, 160
Immonen-Charalambous, K. 144

Inamdar, A. 170

Jungwirth, R. 137

Lawler, M. 132, 144

Lesteven, D. 137

Leyens, L. 187

Moch, H. 160

Negrouk, A. 160

Nutt, D. 170

Ott, M. 137

Pasmans, R. 137

Penault-Llorca, F. 160

Perche, O. 137

Pfister, S.M. 170

Plun-Favreau, J. 144

Pochet, R. 170

Postulka, A. 137
Riegman, P.H.J. 160

Rouzier, R. 144

Schäfer, B. 137

Schneider, D. 160

Schröder-Bäck, P. 178

Souliotis, K. 153

Steuten, L. 144

Strootker, A. 144

Sullivan, R. 132

van den Bent, M.J. 170

Van Hoylandt, N. 170

Weller, M. 170

Westphal, M. 170

Wick, W. 170

Williams, D.-A. 137

Wurcel, V. 137

Zatloukal, K. 160

\section{Subject Index Vol. 19, No. 3, 2016}

Access to health care 153

Adaptive pathways 187

Archived sample 160

Austerity 178

Biobanks 160

Biomarkers 160

Biospecimen 160

Brain banking 170

- disorders 170

Cancer care 132

Capability approach 178

Companion diagnostics 137

Costs of cancer care 132

Diagnostic tests 137

Early dialogue 187

Equitable access 137
Ethics 178

Europe 144

European Medicines Agency 187

Food and Drug Administration 187

Genetic markers 170

Health policy 153

- technology assessment 144, 187

In vitro diagnostics 137

- Individualised therapy 170

Informed consent 160

Innovation 178

Innovative medicines 187

Liquid biopsy 160

Marketing authorization application 187

Measurement 153

Molecular diagnostics 144, 160
Neuromedicine 170

Next-generation sequencing 160

Patient access 144

- involvement 170

Personalised and precision medicine 132

- health care 137

- medicine 137, 144, 178

Personalized medicine 160

Policy 144

Rationalisation 178

Reimbursement 144

- challenges 137

Stakeholders 153

Sustainable health systems 137

Value of diagnostic information 137 